\title{
Placenta accreta: a retrospective study of risk factors, maternal and fetal outcomes in a tertiary hospital in Puerto Rico
}

\begin{abstract}
Introduction: The reported incidence of placenta accreta has increased 10 fold. Cesarean section has been described as one of the most prevalent risk factors. In 2012, the CDC reported a C-section rate of $48.5 \%$ in Puerto Rico (PR). Placenta accreta has been implicated to increase morbidity/mortality in the maternal population as well as to the fetus. The objective of the current study is to describe the population and outcomes of Puerto Rican women diagnosed with abnormal placentation in a tertiary hospital in Puerto Rico.
\end{abstract}

Methodology: We performed a retrospective descriptive study of medical records identified with an abnormal placentation from 2009-2015. The records were obtained from the record room of the University District Hospital of Centro Medico of PR. Data regarding patient demographics, risk factors, maternal and fetal outcomes were recorded using EPI INFO statistical software.

Results: A total of 20 medical records were analyzed. The mean maternal age was 31 years, mean gravity 3.8 and mean gestational age at delivery was 28 weeks. All but one had previous cesarean sections and 1 patient had more than 4 previous cesarean sections. Mean neonatal weight was of $2417 \mathrm{~g}$. 11 patients had an estimated blood loss of more than $2 \mathrm{~L}$ and 10 patients received post-operative blood transfusions. 11 bladder injuries were reported and 17 patients had cesarean hysterectomies performed.

Conclusions: We emphasize the importance of evaluating risk factors and recommend management of suspected cases of abnormal placentation to be done in an interdisciplinary manner and in settingwhere immediate access to blood transfusions is available.

Keywords: placenta accrete, abnormal placentation, interdisciplinary care, bleeding
Volume 2 Issue 4 - 2017

\author{
Gonzalez-Mendez Cristina, Rivera Nelly, \\ Vargas, Darlene, Mendez Keimari \\ Department of Obstetrics and Gynecology, University of Puerto \\ Rico, Puerto Rico
}

Correspondence: Keimari Mendez, Department of Obstetrics and Gynecology, University of Puerto Rico, M6 Calle Clavel, Parques de Santa Maria, San Juan Puerto Rico, Email keimari.mendez@upr.edu

Received: May 16, 2017 | Published: June 09, 2017

\section{Introduction}

Placenta accreta occurs when the deciduas basal is that separates the placental villi from the myometrium is missing. Classification is divided in 3 grades based on histopathologic analysis consisting on: placenta accreta, increate and percent. Placenta accreta occurs when the chorionic villi of the placenta enters in contact with the myometrium. In placenta increta, the chorionic villi invade the myometrium and in placenta percreta the chorionic villi penetrate the uterine serosa. The reported incidence of placenta accreta has increased 10 fold, occurring in about 1:533 pregnanciesfor the period of 1982-2002. Among patients with a histological diagnosis of abnormal placental invasion, $81.6 \%$ of cases were placenta accreta, $11.8 \%$ were placenta increta, and $6.6 \%$ were placenta percreta. ${ }^{1}$ This increase in incidence has been attributed to the prevalence of previously described risk factors, being previous cesarean section one of the most important. ${ }^{2}$

Placenta Accreta has been implicated to increase morbidity/ mortality in the maternal population with detrimental outcomes to the patient as well as to the fetus. It has become one of the leading causes for emergency hysterectomy worldwide accounting for a $51.1 \%$ of emergency hysterectomies. In addition, it is associated with a maternal morbidity in $60 \%$ of cases and a mortality of up to $7 \%$ of cases. ${ }^{3-5}$ Life threatening bleeding is the most common complication. The average blood loss reported at the time of delivery is between
$3000-5500 \mathrm{~mL}$, which leads to significant postoperative morbidity and mortality. ${ }^{6}$ Its early recognition and diagnosis are one of the main goals of management.

Management of placenta accreta has been described as one that should be multidisciplinary involving an obstetrician, interventional radiology, neonatologist, anesthesia service and the creation of protocols for management has been described at different centers with improved outcomes. ${ }^{7}$

In 2012 the CDC reported that a $48.5 \%$ of Puerto Rican babies were delivered by $\mathrm{C}$-section, among the highest rates in the world. Since it has been extensively described that one of the most important risk factor for the development of placenta accreta is a previous cesarean section, ${ }^{8}$ we have confronted a recent increase in this diagnosis. However, there is a gap of information about the incidence, risk factors and outcomes in the population diagnosed with placenta accreta in Puerto Rico, making our study an important step to address this health disparity in our population. Our center is the only tertiary hospital in the island of Puerto Rico were high risk, or suspected high risk obstetrical cases are transferred daily for further management. Due to this finding, this retrospective descriptive study in our center can be considered a representative sample of the population of Puerto Rican patients with placenta accrete and establish referent characteristics for identification of high risk patients with abnormal placentation. 


\section{Materials and methods}

We performed a retrospective descriptive study of medical records identified with an abnormal placentation diagnosis from 20092015. All cases with a presumptive diagnosis of placenta accreta on admission and those with a diagnosis given later during the admission were included. There were no exclusion criteria. Protocol was approved by Medical Sciences Campus IRB, and permission obtained by medical director of the University District Hospital for chart review. Research was conducted according to the principles of the Declaration of Helsinki. The records were obtained from the record room of the University District Hospital in Centro Medico Puerto Rico. Codes for search included ICD9 codes for postpartum hemorrhage, needed blood transfusions, abnormal placentation and intensive care unit admission. A case number was given to each record analyzed to maintain patient confidentiality and was the only identifier used. Data regarding patient demographics, risk factors, maternal and fetal outcomes were obtained from the records and recorded in an electronic database using EPI INFO 7 statistical software, in a one step fashion to eliminate errors during data transfer. Descriptive statistics performed using EPI INFO 7. Primary outcomes of the study is to describe the population of patients diagnosed with placenta accreta to define better the characteristics for identification of high risk patients. Secondary outcomes are to identify management steps that help in the diagnosis and decrease morbidity in the Puerto Rican population diagnosed with abnormal placentation.

\section{Results and discussion}

A total of 20 medical records were analyzed. All were included due to suspected abnormal placentation diagnosis given before delivery. Pre operatively, placentation was described as low lying $(\mathrm{n}=1)$, marginal $(n=1)$, normal $(n=2)$, accrete $(n=5)$, percreta $(n=3)$ and previa $(n=8)$. The mean maternal age was 31years, mean gravity was 3.8 and mean parity was 2 , mean BMI was 28 , and the mean gestational age at delivery was 28 weeks. Nineteen out of 20 had previous cesarean sections, 1 patient had more than 4 previous cesarean sections, but most had only 1 or 2 previous cesarean sections. Five women were smokers $(73 \%), 10$ women reported history of vaginal bleeding $(50 \%)$ and 4 women had history of uterine curettage $(78.9 \%)$

Mean neonatal weight was of $2417 \mathrm{~g}$, mean APGAR of 5 at $1 \mathrm{~min}$ and 7 at 5minutes. NALS had to be performed on 6 neonates and 1 neonatal death was reported. Seven of the babies had to be admitted to NICU (35\% NICU admission).

Thirteen patients had evidence of abnormal placentation on sonography and 4 had evidence of abnormal placentation on MRI. Eleven patients had to be hospitalized prior delivery, on 6 cases hemorrhage protocol had to be implemented and 12 patients received blood transfusions intra operatively. Four patients had placement of internal iliac ballon placement for hemorrhage control (by interventional radiology service) and 11 of the cases were delivered emergently.

Eleven patients had an estimated blood loss of more than $2 \mathrm{~L}$ and 10 patients received post-operative blood transfusions. Two patients had to be admitted to the ICU, and 1 patient received ACLS and died. Eleven bladder injuries were reported (55\% of cases) and 17 out of 20 patients had cesarean hysterectomies performed. The mean hospital stay was of 4 days and the longest was 8 days.
Final pathology results were as follow: normal placentation $(\mathrm{n}=1)$, accretes $(n=9)$, percretas $(n=5)$ and increta $(n=2)$. Comparing variables to final pathology result we obtain certain valuable information. The only maternal death reported had a final pathologic result of a placenta percreta. Two patients with a final pathologic report of placenta percreta $(2 / 5)$ had an EBL of more than $2 \mathrm{~L}, 2$ received post-operative blood transfusions, and 2 received intra operative blood transfusions. One patient with placenta percreta had to be admitted to the ICU. There were 4 cases of placenta percreta with bladder injuries but there were no bowel injuries reported (Figure 1) (Figure 2).

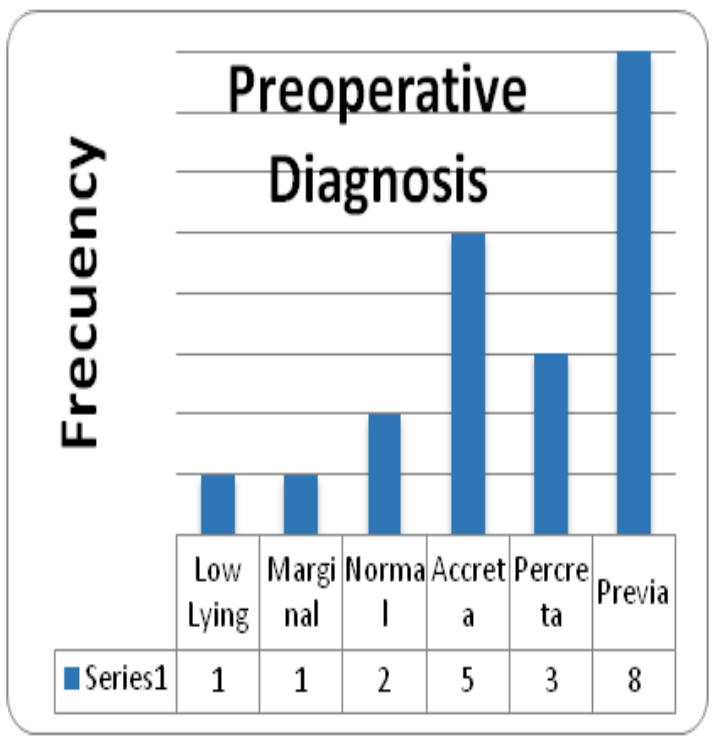

Figure I Preoperative diagnosis.

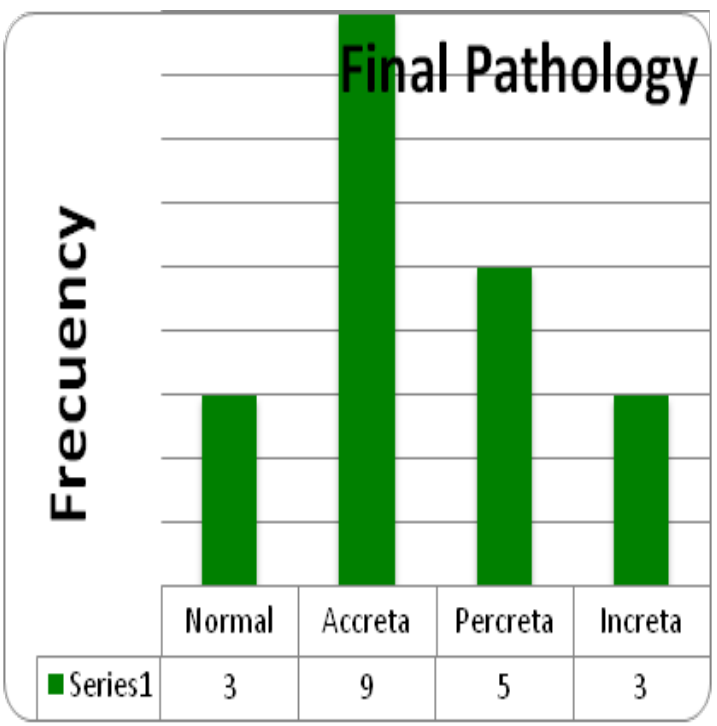

Figure 2 Final pathology results.

Six of the patients (6/9) had a final pathologic report of placenta accreta with an EBL of more than $2 \mathrm{~L}, 7$ received intraoperative blood transfusions and 4 patients received post-operative blood transfusions. Three bladder injuries were reported with this type of placentation also. No bowel injuries were reported. From the 2 patients with final pathologic report of placenta increta, both had an EBL of more than $2 \mathrm{~L}$, received post op blood transfusions and also had intraoperative blood 
transfusions. Only 1 of them had an associated bladder injury and no bowel injuries were reported. From the 17 cesarean hysterectomies that were performed, only 1 had a final pathologic result of normal placentation.

Six patients had an MRI performed. From the 4 cases that MRI predicted abnormal placentation suggesting invasion, 3 resulted in placenta percreta and the other in accreta. The other 2cases that MRI did not detected an abnormality, one had normal placenta pathology and the other one was placenta increta. This difference was not statistically significant, possibly due to the small number of cases that does not allow us to see a clear association. From the 11 patients that had sonographic evidence of abnormal placentation suggesting invasion pre-operatively, 11 had pathologic confirmation of placenta accrete ( 7 cases) and percreta ( 4 cases). On the other hand, from the 4cases where abnormal placentation was not suspected pre operatively, 3 had pathological diagnosis of accrete, percreta or increta, 1 case each. These results had a marginal statistically significant difference with an OR1.8 (0.12-27.8) and p value of 0.58 .

From 16 neonates born to mothers whose final pathologic result was a placenta accreta, increta or percreta, 6 needed NALS compared to 0 out of 3 with normal placentation. This difference was not statistically significant ( $\mathrm{p}$ value 0.3 )

\section{Conclusion}

The main focus of the present study was to describe the population diagnosed with abnormal placentation in a tertiary hospital in Puerto Rico in order to detect variables that may predict adverse maternal and neonatal outcomes. This will help us guide our management to be more aggressive in preventive strategies preoperatively and intraoperatively with those patients with suspected invasion of placenta: accrete increta or percreta.We found that all patients were multiparous and the majority had more than 2 previous cesarean sections. As described by ACOG having multiple cesarean sections increases overall morbidity, not only the one associated to abnormal placentation, but as well as increasing the risk of cystotomy, bowel injury, ureteral injury, admission to the ICU, increase in hospital stay and operative time. ${ }^{11}$ All of these variables were described in this study. The adverse event most commonly reported in our study was cystotomy, mostly associated to placentas percreta. Seventeen of the 20 patients had a cesarean hysterectomy performed at the time of surgery and all but one had a confirmed pathological diagnosis of abnormal placentation. Cesarean hysterectomies are procedures associated to higher morbidity, but in cases of abnormal placentation such procedure may be necessary to save the patient life. In addition to the diagnosis we are studying, the procedure itself is associated to increased blood loss, reason for which most patients in our study had an EBL more than $2 \mathrm{~L}$, with 12 patient needing intra operative blood transfusions. This implies abnormal placentation cases should be managed in a hospital setting where immediate blood transfusions as well as intra operative consults can be made in an interdisciplinary manner (Table 1) (Table 2)

In our study we identified that 11 of these cases where delivered emergently. This poses a bigger risk for the patient since pre-operative preparation cannot be made with consults (urology, interventional radiology, neonatology, MFM) and availability of blood bank. Our study implies the importance of having at least the awareness of the possibility of an abnormal placentation by describing demographics and risk factors of the population of Puerto Rico, thus emphasizing the importance of our study.
Table I Demographics variables

\begin{tabular}{lll}
\hline Variable & Median & SD \\
\hline Age & 31.2 & 6.88 \\
Grava & 3.8 & 1.73 \\
Para & 2 & 0.97 \\
Bmi & 28.31 & 4.2 \\
Abortion & 0.8 & 1.4 \\
Gestational Age & 28.8 & 7.68 \\
\hline
\end{tabular}

Table 2 Abbreviations: HX, History

\begin{tabular}{llll}
\hline Variable & Yes & No & Percentage \\
\hline Smoking & 5 & 14 & $26 \% / 73 \%$ \\
Uterine Anomalies & 0 & 20 & $0 \% / 100 \%$ \\
HXVaginal Bleeding & 10 & 10 & $50 \% / 50 \%$ \\
HX of Uterine Curretage & 4 & 15 & $21 \% / 78.9 \%$ \\
HX of Uterine Radiation & 0 & 20 & $0 \% / 100 \%$
\end{tabular}

Imaging studies help plan the course of management and, as previously stated, had help to minimize the morbidity associated with abnormal placentation. In our study we were able to confirm a positive association of sonographic evidence of an abnormal placentation with a pathological diagnosis of placenta accrete, percreta or increta, with an OR 1.8. The mean gestational age of delivery was 28 weeks and most cases were performed emergently. This explains our study's high rate of neonates requiring NICU admission, regardless of final pathological result of placentation. Our results are in concordance with the fact that the diagnosis of abnormal placentation is associated to neonatal morbidity mostly link to prematurity.

One of the limitations of the present study is the unavailability of electronic medical record which increases the possibility that not all medical records of abnormal placentation were retrieved and limits the quality of information obtained.

In conclusion most of the described patients with abnormal placentation were multiparous and had 2 or more previous cesarean sections. Most of the patients had an EBL of more than $2 \mathrm{~L}$ and cystotomy was the adverse event most commonly reported. The prenatal care and delivery should be done in a setting where immediate access to blood transfusions and subspecialist is available, given the high volume of blood transfusions needed and high rate of bladder injuries that merits the intervention of urologists during the same surgery, all done to ensure the best outcome possible to the patients.

The high morbidity associated with a diagnosis of an abnormal placentation has been extensively described in the literature and now with this study is also described in a Puerto Rican population. Prompt recognition of described risk factors help identifies the highest risk patients in order to facilitate early referral to tertiary centers for complete evaluation and management. In the future, we plan to continue increasing our sample size to provide a more extensive evaluation of management variables that can improve maternal and fetal outcomes in order to create more specific recommendations for the treatment of patients with abnormal placentation in the Puerto Rican population Table 3. 
Table 3 Categorization of variables in the study population

\begin{tabular}{|c|c|c|c|}
\hline \multirow{7}{*}{ Management variables } & \multicolumn{3}{|l|}{ Yes No } \\
\hline & Pre Op Hospitalization in antepartum ward & II (57.8\%) & $8(42.1 \%)$ \\
\hline & Hemorrhage protocol activated & $6(31 \%)$ & $13(68 \%)$ \\
\hline & MRI evidence of Accreta & $4(21 \%)$ & $2(10.5 \%)$ \\
\hline & Sonographic evidence of Accreta & $13(68 \%)$ & $4(2 \mid \%)$ \\
\hline & Uterine Artery Balloon Placement & $4(2 \mid \%)$ & $15(78 \%)$ \\
\hline & Intra Op Blood Transfusion & $12(63 \%)$ & $7(36 \%)$ \\
\hline \multirow{9}{*}{ Maternal outcomes variables } & \multicolumn{3}{|l|}{ Yes No } \\
\hline & Post Op Blood Transfusion & $10(50 \%)$ & $10(50 \%)$ \\
\hline & Maternal Death & I (5\%) & $19(95 \%)$ \\
\hline & ACLS given & $\mathrm{I}(5 \%)$ & $19(95 \%)$ \\
\hline & ICU admission & $2(10.5 \%)$ & 17 (89.4\%) \\
\hline & Bowel Injury & $0(0 \%)$ & $20(100 \%)$ \\
\hline & acute kidney injury & $0(0 \%)$ & $20(100 \%)$ \\
\hline & Bladder Injury & II (55\%) & $9(45 \%)$ \\
\hline & Cesarean Hysterectomy & I7(89\%) & $2(10.5 \%)$ \\
\hline \multirow{4}{*}{ Neonatal outcomes variables } & \multicolumn{3}{|l|}{ Yes No } \\
\hline & NALS given & $6(31.5 \%)$ & I3(68.4\%) \\
\hline & Neonatal Death & $\mathrm{I}(5 \%)$ & $19(95 \%)$ \\
\hline & NICU admission & $7(36.8 \%)$ & $12(63 \%)$ \\
\hline
\end{tabular}

Pre Op, pre operative, Intra Op, intra operative, Post Op, post-operative, ACLS, advaced cardiac life support, ICU, intensive care unit, NALS, neonatal advanced life support, NICU, neonatal intensive care unit

\section{Acknowledgements}

None.

\section{Conflict of interest}

The views expressed in this article are original research, no funding received, no external sources of bias. No other sources of support, grants or equipment received in the performance of this study. No conflict of interests to report.

\section{References}

1. Morlando M, Sarno L, Napolitano R, et al. Placenta Accreta:incidence and risk factors in ana area with a particularly high rate of cesarean section. Acta Obstet Gynecol Scand. 2013;92(4):457-460.

2. Eshkoli T, Weintraub AY, Sergienko R, et al. Placenta Accreta: risk factors, perinatal outcomes, and consequences for subsequent births. $\mathrm{Am} \mathrm{J}$ Obstet Gynecol. 2013;208:219e1-231e7.
3. Angstmann T, Gard G, Harrington T, et al. Surgical management of placenta accrete: a cohort series and suggested approach. Am J Obstet Gunecol. 2010;202(1):38e1-38e9.

4. Orbach A, Levy A, Wiznitzer A, et al. Peripartum cesarean hysterectomy: critical analysis of risk factors and trends over the years. J Matern Fetal Neonatal Med. 2011;24(3):480-484.

5. Sumigama S, Itakura A, Ota T, et al. Placenta previaincreta/percreta in Japan: a retrospective study of ultrasound findings, magement and clinical course. J Obstet Gynaecol Res. 2007;33(5):606-611.

6. Eller AG, Porter TT, Soisson P, et al. Optimal management strategies for placenta accreta. BJOG. 2009;116(5):648-654.

7. Garmi G, Salim R. Epidemiology, etiology, diagnosis and management of placenta accrete. Obstet Gynecol Int. 2012;2012:873929.

8. Wong HS, Cheung YK, Zuccollo J, et al. Evaluation of sonographic diagnostic criteria for placenta accrete. J Clin Ultrasound. 2008;36(9):551-559. 